\title{
Microscopic Character and Movement Consistency of Pedestrian Group: An Experimental Study in Campus
}

\author{
XIAOGE WEI, XU MAI, WEI LV, WEIGUO SONG \\ State Key Laboratory of Fire Science \\ University of Science and Technology of China \\ Hefei 230026, PR China
}

\begin{abstract}
The research of human crowd dynamic is significant for the building designers and emergency guiders. However, current researches mainly focus on the interactions among isolated individuals, the influence of social interactions among pedestrian is needed to take into consideration in the pedestrian dynamic study. In this manuscript we carried out observed experiments of the movement of pedestrian group and used optical flow algorithm to extract pedestrian's trajectory from the video. Then the movement characteristics of the group members were analyzed and discussed, which include the angle, the distance, the velocity, the group alignment, step frequency and the offset angle of group member. It indicates group members have adaptive ability to maintain the group structure and the velocity fluctuation of the group is small. It is found that the velocity and step frequency of group member are smaller than that of individuals under the same scenario. Movement consistency of the group members is also verified. These experimental results will be a support for modeling pedestrian group.
\end{abstract}

KEYWORDS: evacuation, pedestrian dynamic, group behavior, experiment, consistency.

\section{INTRODUCTION}

With the increasing population and economy growth in cities, there is an urgent problem to evacuate pedestrian in public places with high crowd density. Thus the research on the pedestrian evacuation and behavior has widely drew many researchers' attention. On the whole, there are two basic methods, modeling and experiments. With the last decade research, both the evacuation modeling [1] and experiment research [2] have a rapid development. The researchers find many typical phenomenons such as arching around bottlenecks [3], the segregation of opposite flows [4] or the turbulent movement in high dense crowds [5], the 'faster is slower' [6] and so on. But they are about the 'physics' of crowd motion. However, only a few modeling study and experiment research has considered the impact of social interactions. Indeed, the great majority of the previous studies focused on the movement of isolated individuals. In fact, it turns out that the majority of pedestrians actually do not walk alone, but they walk with friends, relatives or someone else [7-10].

For the researchers of studying crowd dynamics, the pedestrian group research still is in the initial phase. The term 'group' is used here in its sociological sense $[7,11]$, that is, not only referring to several proximate pedestrians that happen to walk close to each other, but to individuals who have social ties and intentionally walk together, such as friends or family members. Thus the research on pedestrian group has been widely researched by the sociologist and psychologists. They paid much attention to how the social factors influence on the pedestrian group movement qualitatively. Currently the research on the pedestrian group mainly focus on several aspects, including the group size $[12,13]$, the group composition, the group formation, the group member's orientation, interpersonal distance [14]. Actually the great majority of group researches still are under the stage of these proxemic variables study. In fact, there are many effect factors should not be neglected, such as dominance, attraction, age, similarity, gender, power, status, cross-cultural, personality determinants, the city size [15-17] and so on.

Harmeet [18] investigated the behavior of subgroups in crowd dynamics by means of filming and observation. Then they modified a discrete element model by incorporating psychological forces and realistic subgroup behavior. Musse et al. [19] presented a new approach of crowd behavior considering the relationship between groups of individuals and the emergent behavior originated from it. In addition they described a multiresolution collision method specific for the crowd modeling. Marco Costa [20] observed 1,020 groups in an urban environment, and they used the statistic analysis method to investigate the group alignment. They found that walking speed was higher in all-male groups. In addition, they stressed that walking speed was slowest in mixed dyads because of a deeper reciprocal involvement. Moussaïd [7] analyzed the motion of approximately 1500 pedestrian groups under natural condition, and obtained typical group walking patterns in different crowd density. On the basis of the statistic data, they improved the social force model by considering the effect of the present of pedestrian groups. Qiu [21] presented a unified and well defined framework for modeling the structure aspect of different groups in pedestrian crowds by considering both intra-group 
structure and inter-group relationships. Based on this framework, an agent-based crowd simulation system was developed and crowd behavior simulation was exhibited. Wang [22] proposed a cellular automation model to simulate team-moving behavior in bidirectional pedestrian flow. Then they put forward four movement patterns of double-pedestrian teaming. Simulation results showed that team-moving produced significant corridor capacity effect, and such effects highly depended on the type of teaming behavior. On the basis of the conventional social force model, Guo [23] studied communicative crowd motion occurs under the impact of stores. The members consequently formed a trade-off between communicating with other team members and browsing stores on street side. On the whole, with regard to the pedestrian group dynamics, most research rested on modeling, which also needs empirical data to validate. Thus carrying out the experiment of the pedestrian group movement is important and meaningful.

Compared with the experiment research on single pedestrian, the research on pedestrian group is faced with some challenges. The most important one is that it is hard to get the effective data of pedestrian group movement by the control laboratory experiment. What's more, it is inevitable that the social relationship among pedestrians affects pedestrian movement. Taking the above factor into consideration comprehensively, the observed experiment is a good measure to study pedestrian group movement. In this work, an observed experiment was carried out in a university campus to investigate the pedestrian group movement.

\section{EXPERIMENTAL SETUP}

The observed experiment was carried out in March 22, 2013 and the highest temperature of that day was about 10 Celsius, which was low temperature, so people wore quite thick clothing. The video shooting time was from 17:00 to 18:00, which is the dinner time for the students. The observed place was performed in a 4-meter-width passageway in the campus of university of science and technology. Figure 1 shows a diagram of the scenario. In view of the fact that this passage is an important channel towards the school canteen, thus groups of students walk together along this passage in the meal time. For most part of students, they spend a lot of time studying or working with their friends or partners. Therefore it is easy to observe the cases pedestrians walking in group. The students go in the observation area from line $\mathrm{CD}$ (or $\mathrm{AB}$ ) and leave at line $\mathrm{AB}$ (or $\mathrm{CD}$ ). In order to specify the equations in the next content, here we marked two coordinates. The participants in this observed experiment are students, aging from 20 to 25, and most people's height is about $160-180 \mathrm{~cm}$. The reason why we chose the students as the observed sample was that their social relationship was clear and simple. Their status is equal and usual behavior is similar, so the effect of social factor is light and can be ignored. In addition a video camera with a resolution of $1440 * 1080$ was located on the seventh floor, approximately $20 \mathrm{~m}$ above the ground. Because the camera is not possible to mount just above the measurement area, we adopted four known points (A, B, C and D) to adjust the distortion caused by lens distortion using direct linear transformation (DLT) [24], as shown in Fig.1. The perspective distortion effect caused by the different heights of the participants is inevitable and has to be ignored.

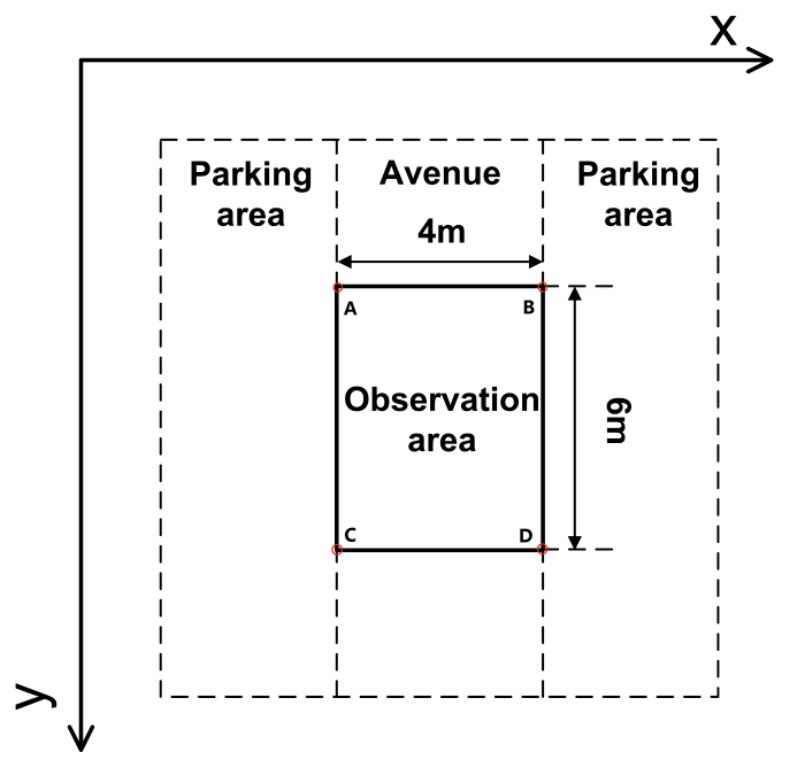

Fig. 1. Schematic diagram of the observed scene.

Because the school is a science technology university, the number of male student is much larger than female. Here we mainly focus on male students. One of benefits is easy to obtain observed samples, and the other is to exclude the effect of gender 
difference. According to the previous research, we have known that the surrounding density of pedestrian group affects pedestrian's velocity, personal space and formation. In this manuscript, our aim is to research the inner movement rules of pedestrian group, meantime reduce the interference from the outside factors as far as possible. Thus we choose the male pedestrian group in the freely walking circumstance as the observed sample. Here the freely walking circumstance means that there is no other pedestrian walking in their surrounding when one pedestrian group walk in the observed region. After getting the experiment video, every pedestrian's location could be obtained by the tracking algorithm and image distortion calibration algorithm. Furthermore, pedestrian's walking velocity, interpersonal distance, offset angle was computed.

\section{TRACKING ALGORITHM}

In this observed experiment, we used the optical flow algorithm to extract pedestrian's trajectory. The basic principle of optical flow method for detecting moving objects is presented as follows. A velocity vector is given for each pixel for one image, and there will generate motion field of the image. The pixel point of the image and the true 3D object has a one-to-one correspondence at a particular moment, which can be obtained by the projection relation. The image can be analyzed dynamically, according to the characteristics of each pixel's velocity vector. If there is no moving object in the image, the optical flow vector is continuously changing in the whole image. When there is a moving object in the image, the velocity vector formed by the moving object is different from the one generated the close-by background. Using this method, we can detect the moving object and get its position. The optical flow method to detect moving objects has many advantages, which not only carries the movement information of the object but also contains plenty of 3D structure information. What's more this method can detect the motion object without knowing any information about scene.

\section{RESULTS AND ANALYSIS}

In this manuscript, we chose 20 groups of male dyads, 10 groups of male triads, 8 groups of four male pedestrians and 30 groups with single male pedestrian. All these observed groups are in the freely walking circumstance, and their movement is basically not affected by the outside factors.

\section{Average Angle and Distance between Group Members}

In order to investigate the pedestrian group movement pattern, we refer to the method in [7]. We define $\alpha_{i j}$ and $d_{i j}$ as the angle and distance between pedestrians $\mathrm{i}$ and $\mathrm{j}$, where $\mathrm{i}$ and $\mathrm{j}$ belong to the same group and $\mathrm{j}$ is its closest neighbor on the right-hand side.

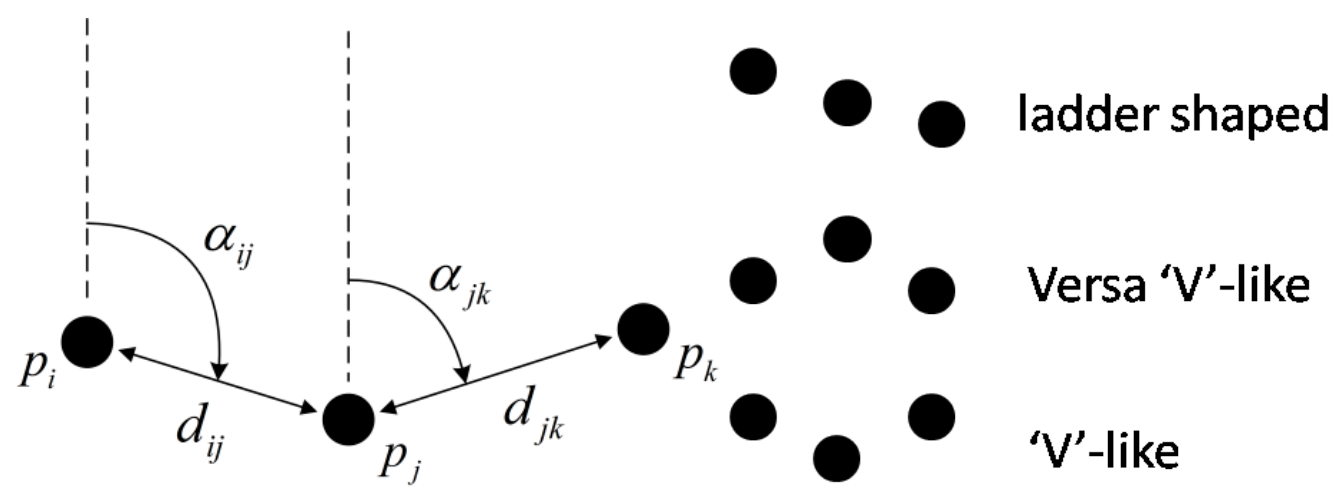

(a)

(b)

Fig. 2. (a) Illustration of the measurement method. (b) Different group formation structure.

Table 1. Average angle and distance values between group members for each group size

\begin{tabular}{|c|c|c|c|}
\hline Group size & Neighbor members & $\mathbf{d}_{\mathbf{i j}}(\mathbf{m})$ & $\boldsymbol{\alpha}_{\mathbf{i j}}(\mathbf{d e g})$ \\
\hline Size $=2$ & $\mathrm{p}_{1} \mathrm{p}_{2}$ & $0.68( \pm 0.16)$ & $89.20( \pm 15.36)$ \\
\hline Size $=3$ & $\mathrm{p}_{1} \mathrm{p}_{2}$ & $0.57( \pm 0.10)$ & $102.07( \pm 20.05)$ \\
\hline & $\mathrm{p}_{2} \mathrm{p}_{3}$ & $0.56( \pm 0.19)$ & $69.00( \pm 20.05)$ \\
\hline Size $=4$ & $\mathrm{p}_{1} \mathrm{p}_{2}$ & $0.61( \pm 0.10)$ & $110.41( \pm 15.17)$ \\
\hline & $\mathrm{p}_{2} \mathrm{p}_{3}$ & $0.58( \pm 0.10)$ & $91.99( \pm 30.03)$ \\
\hline & $\mathrm{p}_{3} \mathrm{p}_{4}$ & $0.57( \pm 0.08)$ & $73.21( \pm 23.61)$ \\
\hline
\end{tabular}


Table 2. Average angle and distance values between group members for each group size and density level [7].

\begin{tabular}{|c|c|c|c|c|c|}
\hline & & \multicolumn{2}{|c|}{ Population A (low density) } & \multicolumn{2}{c|}{ Population B (high density) } \\
\hline Group size & Neighbor members & $\mathbf{d}_{\mathbf{i j}}(\mathbf{m})$ & $\boldsymbol{\alpha}_{\mathbf{i j}}(\mathbf{d e g})$ & $\mathbf{d}_{\mathbf{i j}}(\mathbf{m})$ & $\boldsymbol{\alpha}_{\mathbf{i j}}(\mathbf{d e g})$ \\
\hline Size=2 & $\mathrm{p}_{1} \mathrm{p}_{2}$ & $0.78( \pm 0.02)$ & $89.8( \pm 1.12)$ & $0.54( \pm 0.01)$ & $90.3( \pm 0.80)$ \\
\hline Size$=3$ & $\mathrm{p}_{1} \mathrm{p}_{2}$ & $0.79( \pm 0.05)$ & $97.8( \pm 5.14)$ & $0.55( \pm 0.01)$ & $107.9( \pm 2.84)$ \\
\hline & $\mathrm{p}_{2} \mathrm{p}_{3}$ & $0.81( \pm 0.10)$ & $87.1( \pm 4.46)$ & $0.62( \pm 0.04)$ & $70.6( \pm 2.55)$ \\
\hline Size=4 & $\mathrm{p}_{1} \mathrm{p}_{2}$ & $0.87( \pm 0.06)$ & $99.2( \pm 6.33)$ & $0.67( \pm 0.02)$ & $102.3( \pm 5.85)$ \\
\hline & $\mathrm{p}_{2} \mathrm{p}_{3}$ & $0.93( \pm 0.09)$ & $87.7( \pm 6.54)$ & $0.66( \pm 0.02)$ & $86.0( \pm 4.71)$ \\
\hline & $\mathrm{p}_{3} \mathrm{p}_{4}$ & $0.80( \pm 0.05)$ & $85.4( \pm 5.01)$ & $0.64( \pm 0.03)$ & $76.6( \pm 5.09)$ \\
\hline
\end{tabular}

Note: Values between brackets indicate the standard error of the mean.

In order to compare with Moussaïd's results, table 2 was also exhibited. Therefore short introduction of Moussaïd's work is necessary. Moussaïd'data mainly has two parts, populations A (low density) and B (high density). While population A was observed during the afternoon of a working day, population B was observed on a Saturday in a popular commercial walkway [7].

Data in table 1 and table 2 suggest that, in the freely walking circumstance, the average angles are different from the outputs of Moussaïd in low density. And the standard errors in the brackets are much larger than Moussaïd's results. In fact, in the video, except pedestrian walking abreast, we could find that there existed some other group formation structure, such as ladder shaped, 'V'-like and so on, which can be seen in Fig. 2 (b). In addition, maybe the methods to calculate $\alpha_{\mathrm{ij}}$ by us and Moussaïd are different. However, the average distance between group members $\left(\mathrm{d}_{\mathrm{ij}}\right)$ is $0.1 \mathrm{~m}-0.2 \mathrm{~m}$ smaller than that of Moussaïd in low density. The difference between this paper and Moussaïd's could potentially be attributed to three causes. The first is the difference of body size because the calculation of $\mathrm{d}_{\mathrm{ij}}$ contains part of body; the second is the cultural differences between Eastern and Western countries, because different cultures will cause the interpersonal distance's difference; and the third one is the social relationship among group members. In this paper, all the observed pedestrians are college students with the same social status, while Moussaïd observed pedestrians after working. On the other hand, the $\mathrm{d}_{\mathrm{ij}}$ and $\alpha_{\mathrm{ij}}$ we obtained is more consistent with Moussaïd's results in high density. Therefore I suspect that there exists some relationship between $\mathrm{d}_{\mathrm{ij}}$ and $\alpha_{\mathrm{ij}}$. In order to specify the preservation of group formation, for each group the $\mathrm{d}_{\mathrm{ij}}$ and $\alpha_{\mathrm{ij}}$ were recorded in every frame. Then we got the standard error of the mean of each group's $d_{i j}$ and its value was about $0.15 \mathrm{~m}$. Such results indicate that in the movement process group members can maintain the group structure and the group has certain stability. Therefore we heavily analyze how the group members adjust their movement in order to achieve movement consistency of the group.

\section{Velocity of Group Member}

Velocity is an intuitional parameter to study pedestrian movement, which is also important for group movement. Fig. 3 illustrates the mean velocities of the observed participants, including single pedestrian and group member. Each pedestrian's velocity can be calculated by equation 1 .

$v(i, t)=\frac{\sqrt{(x(i, t+\Delta t)-x(i, t))^{2}+(y(i, t+\Delta t)-y(i, t))^{2}}}{\Delta t}$

Here $t$ is current frame's index, and $\mathrm{x}(\mathrm{i}, \mathrm{t}), \mathrm{y}(\mathrm{i}, \mathrm{t})$ are the $\mathrm{x}, \mathrm{y}$ coordinates of pedestrian $\mathrm{i}$ in the current picture. $\Delta t$ is equal to 10 , that is to say the time period is $0.33 \mathrm{~s}$. Because there are 30 frames in one second, the time period of one frame is $0.033 \mathrm{~s}$ and that of ten frames is $0.33 \mathrm{~s}$. By each group member's average velocity, we can obtain pedestrian group's average velocity. 


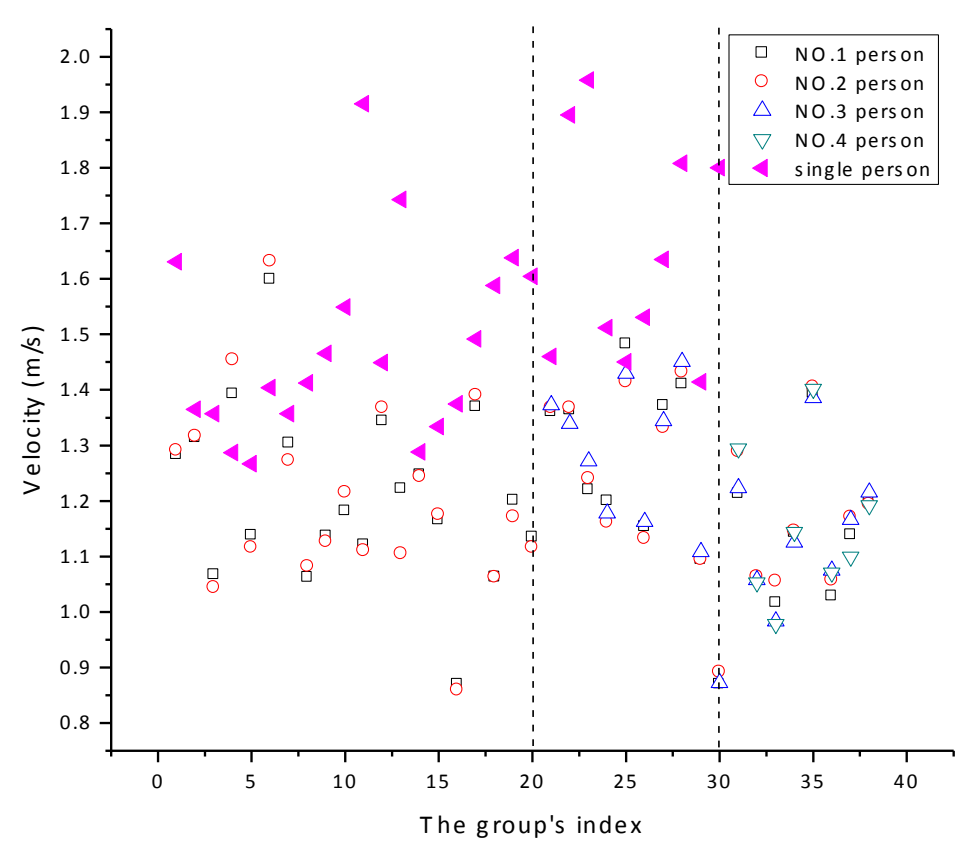

Fig. 3. The velocity of different group. NO.1 person, NO.2 person, NO.3 person and NO.4 person are the first person, second person, third person and fourth person of the group. And 1-20 are the dyads group, 21-30 are the triads group, 31-38 are the 4

persons groups.

Compared with single pedestrian's velocity in the freely walking circumstance, group member's velocity obviously decreases. As Fig. 3 is shown, most of single pedestrian's velocity is higher than $1.25 \mathrm{~m} / \mathrm{s}$, and even some pedestrian's velocity reaches $1.9 \mathrm{~m} / \mathrm{s}$. The average velocity of all the groups with 2 persons or 4 persons is about $1.2 \mathrm{~m} / \mathrm{s}$, while the average velocity of all the groups with 3 persons is $1.3 \mathrm{~m} / \mathrm{s}$. All the pedestrians observed here are in the freely walking circumstance, but when pedestrians walk in group, his velocity will decrease. From this view, it proves that the social factor indeed influences on pedestrian movement. On the whole, for different group size, the average velocity difference is very small. Because each pedestrian group that we observe here walks in a freely walking circumstance, and pedestrian's movement has been little affected by the surrounding environment. Thus the effect of group size towards pedestrian velocity is not very evidence. However, for the same group size, the velocity of different group has some distinct difference. For the dyads group, triads group, 4 persons group, the average velocity differences are $0.8 \mathrm{~m} / \mathrm{s}, 0.6 \mathrm{~m} / \mathrm{s}, 0.4 \mathrm{~m} / \mathrm{s}$ respectively. It reflects that the movement characteristic of different pedestrian is different in the freely walking circumstance. What's more, the larger the group size is, the smaller the velocity difference of different group is. For a certain group, no matter how large the group size is, the group velocity fluctuates near the group's average velocity, and the fluctuation region is about $0.2 \mathrm{~m} / \mathrm{s}$. The small fluctuation proves that pedestrian can have a good control of his velocity.

\section{Group Alignment}

When the researchers study the pedestrian group, they usually adopt alignment level to characterize the movement of pedestrian social group [20]. In the common sense, when the alignment level is better, it can be regarded that the group's cohesive force is large and the group's stability is good. As displayed in Fig. 4, each group member locates in the place with some distance from the group location center, therefore the alignment level is the average of all these distance. Here we use the parameter $\mathrm{P}_{\text {alignment }}$ to measure the alignment level. Using the Eq. (2), Eq. (3) and Eq. (4), $\mathrm{P}_{\text {alignment }}$ can be obtained.

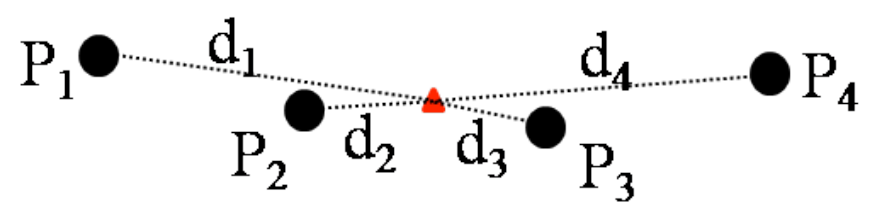

Fig. 4. The black solid circles indicate group members and the red solid triangle expresses the group location center. $\mathrm{P}_{\mathrm{i}}$ represents person $\mathrm{i}$, and $\mathrm{d}_{\mathrm{i}}$ stands for the distance from person $\mathrm{i}$ to the group location center. 


$$
\begin{aligned}
& x_{\text {center }}=\frac{1}{n} \sum_{i=1}^{n} x_{i} \\
& y_{\text {center }}=\frac{1}{n} \sum_{i=1}^{n} y_{i} \\
& p_{\text {alignment }}=\frac{1}{n} \sum_{i=1}^{n} \sqrt{\left(x_{i}-x_{\text {center }}\right)^{2}+\left(y_{i}-y_{\text {center }}\right)^{2}}
\end{aligned}
$$

Where $x_{i}$ is the $x$ coordinate of group member $i, y_{i}$ is the $y$ coordinate of group member $i, x_{\text {center }}$ is the $x$ coordinate of the center point of the group, $\mathrm{y}_{\text {center }}$ is the $\mathrm{y}$ coordinate of the center point of the group.

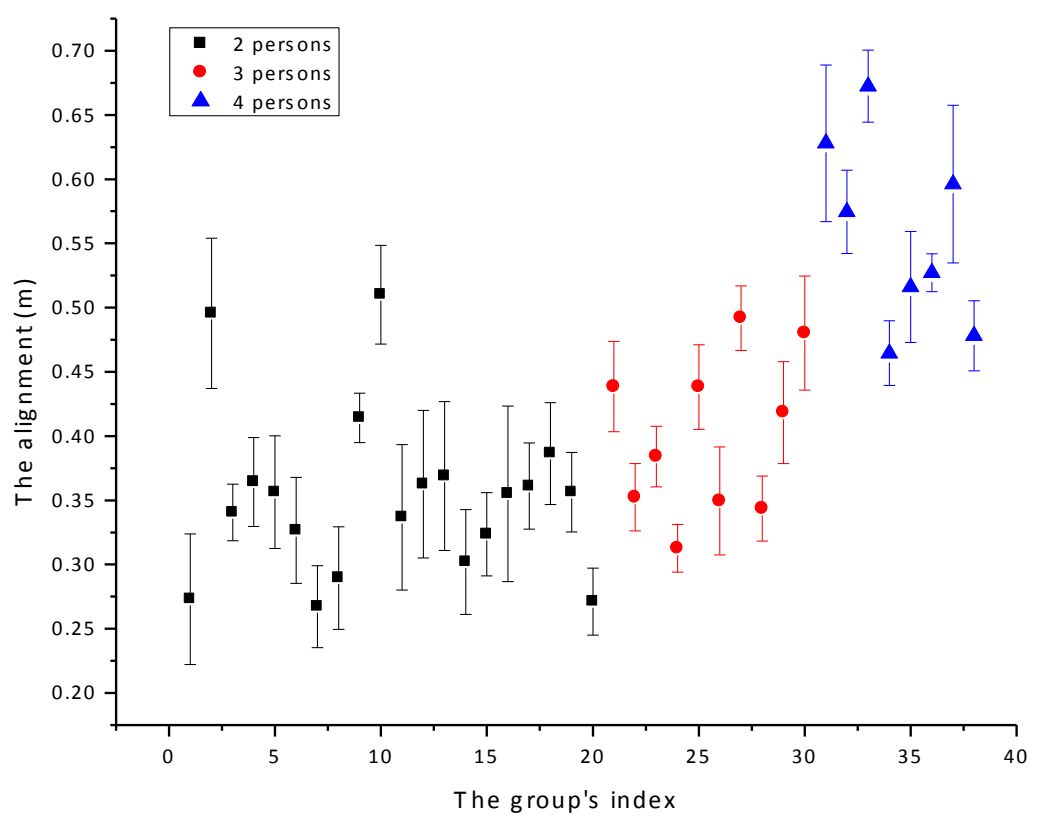

Fig. 5. The alignment level of different group. 1-20 are the dyads group, 21-30 are the triads group, 31-38 are the 4 persons groups.

As indicated in Fig. 5, there exist some obvious differences among the alignment level of different group size. The alignment level of the dyads group is relatively small, and most of them are below $0.35 \mathrm{~m}$. However the alignment of the triads group and four persons group are about $0.4 \mathrm{~m}$ and $0.55 \mathrm{~m}$ respectively. In addition, no matter how large the group size is, if the pedestrian is far away from the center of the group, it will bring inconvenience to communicate with other pedestrian in the same group. Therefore the $\mathrm{P}_{\text {alignment }}$ value will not increase without limit.

\section{Relationship between Density and Velocity}

If we ignore the social factors' effect and regard each pedestrian as a single individual, the other pedestrians in the same group will influence the individual's movement. For a certain pedestrian, owing to other pedestrian's existence in the same group, it will produce density effect to this individual. It means that pedestrians in the same group will affect each other's movement. In order to explore this problem, we want to find the relationship between group density and group velocity. Thus we need a new definition of pedestrian group density, which also can be regarded as the internal density of pedestrian group. The groups we observed were in the freely walking circumstance, no other pedestrian affects the group movement. There was no pedestrian right ahead of each group member, so group member's movement is mainly affected by the lateral pedestrians. Therefore we can use the one dimensional lateral density to express the whole density. Referring to the method of using the headway to define density, we use the closest distance to compute one pedestrian's surrounding density, shown as Eq (5). Owing to the difference of group member's condition, we divided group members into five categories, seen Fig.6. 


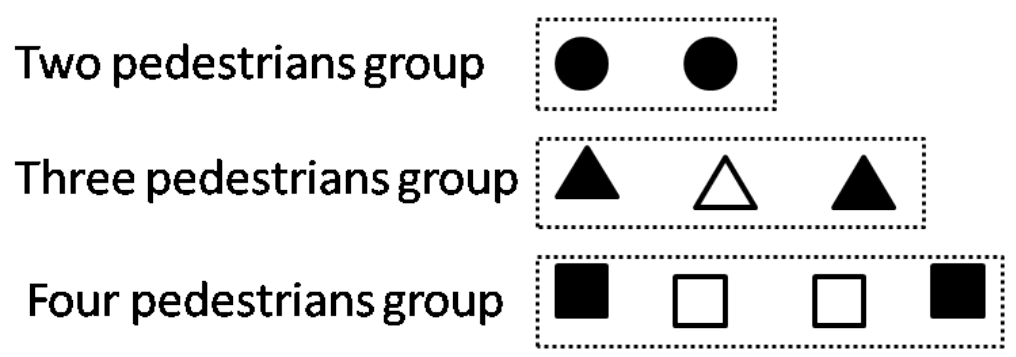

Fig. 6. Five categories of group member. The solid circles, the solid triangles, the solid squares, the hollow triangle and the hollow square are the first, second, third, fourth and fifth category.

$$
\operatorname{densiy}(i)=\frac{1}{\min _{j \in N}\left(d_{i j}\right)}
$$

Here $\mathrm{N}$ is the set of person i's neighbor.

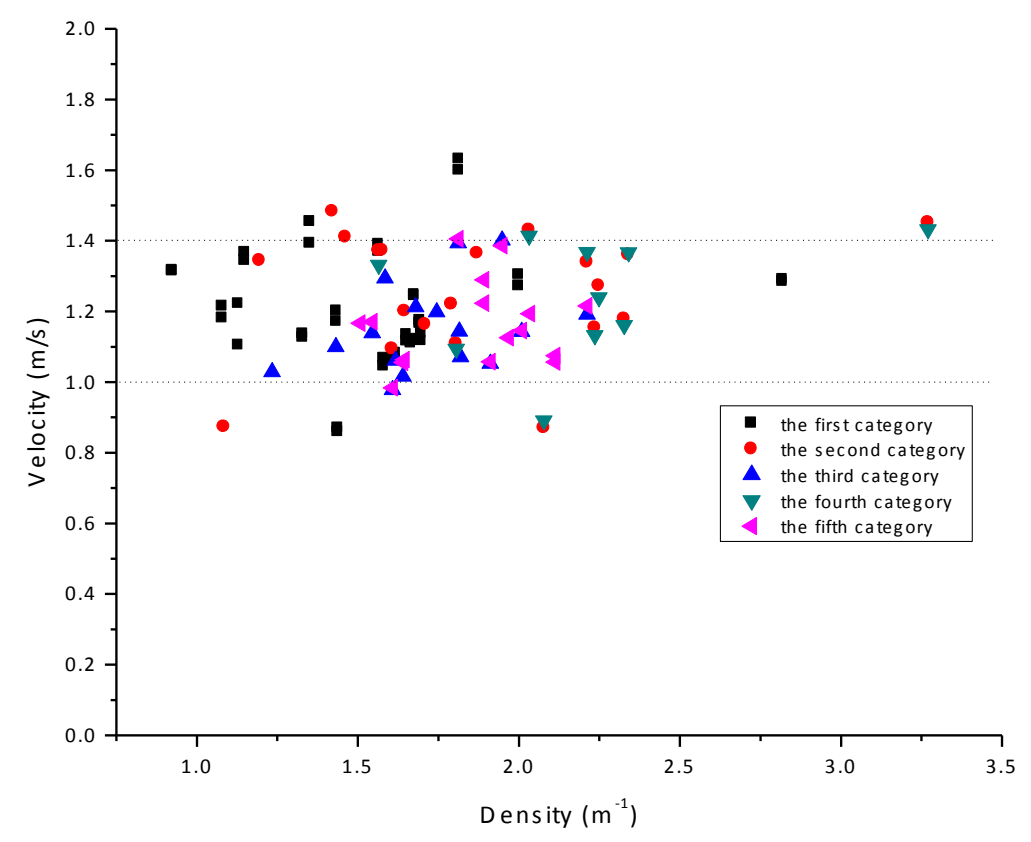

Fig. 7. The density and velocity of different group size

The Chinese's body size is about $0.25 \mathrm{mby} 0.45 \mathrm{~m}$ [25], and the distance between two persons are about $0.45 \mathrm{~m}$ or larger. Thus most of pedestrian's density is smaller than $2.5 \mathrm{~m}^{-1}$. From Fig. 7 , it can be found that with the density's increasing pedestrian's velocity shows little change. What's more the velocity of five categories of pedestrian has no evident difference. Most of pedestrian's velocity fluctuates near $1.2 \mathrm{~m} / \mathrm{s}$. It indicates that the lateral neighbors have little influence on one pedestrian's movement. This result is quite similar with the conclusion in [26], where regarded that lateral interference did not have a strong influence on the fundamental diagram in the considered density range. Thus we can take all the group members as one whole entity, which has the same movement dynamics. On the whole, the law between density and velocity of pedestrian group is more complicated than the situation of pedestrians without group. The rule is influenced by not only the surrounding pedestrian density but also the communication among the group members.

Owing to cohesive force of pedestrian group, it promotes greater coherence in the pedestrians' movement in the same group. Just as we often see, if one pedestrian changes direction, the other pedestrians in the same group will adopt the same movement pattern. This is quite different from the situation that two individuals without social relationship walk abreast. It means that pedestrian group decreases the freedom of pedestrian's movement. In other words, it also proves that the whole pedestrian group has consistency in movement. In order to illustrate the coordination and consistence of group movement, it is important to deeply analyze pedestrian group movement process. 
For a certain pedestrian group, every 10 frames, every pedestrian's instantaneous velocity can be calculated. Furthermore the each pedestrian's velocity evolution process is obtained. Here for different group size, we choose one group's result as illustration. From Fig.8, it can be seen that pedestrian's instantaneous velocity presents the change trend of the sine function. It's the pedestrian's walk pattern causing such result. It is known that, people walk on their two legs with an upright body posture. What's more, the previous researches prove that pedestrian swing in the walk process. Besides pedestrian's one step is a tiny process and there will produce different instantaneous velocity in such a process. No matter how large the group size is, each group's average instantaneous velocity keeps still and the fluctuation is about $0.2 \mathrm{~m} / \mathrm{s}$ near the average value.

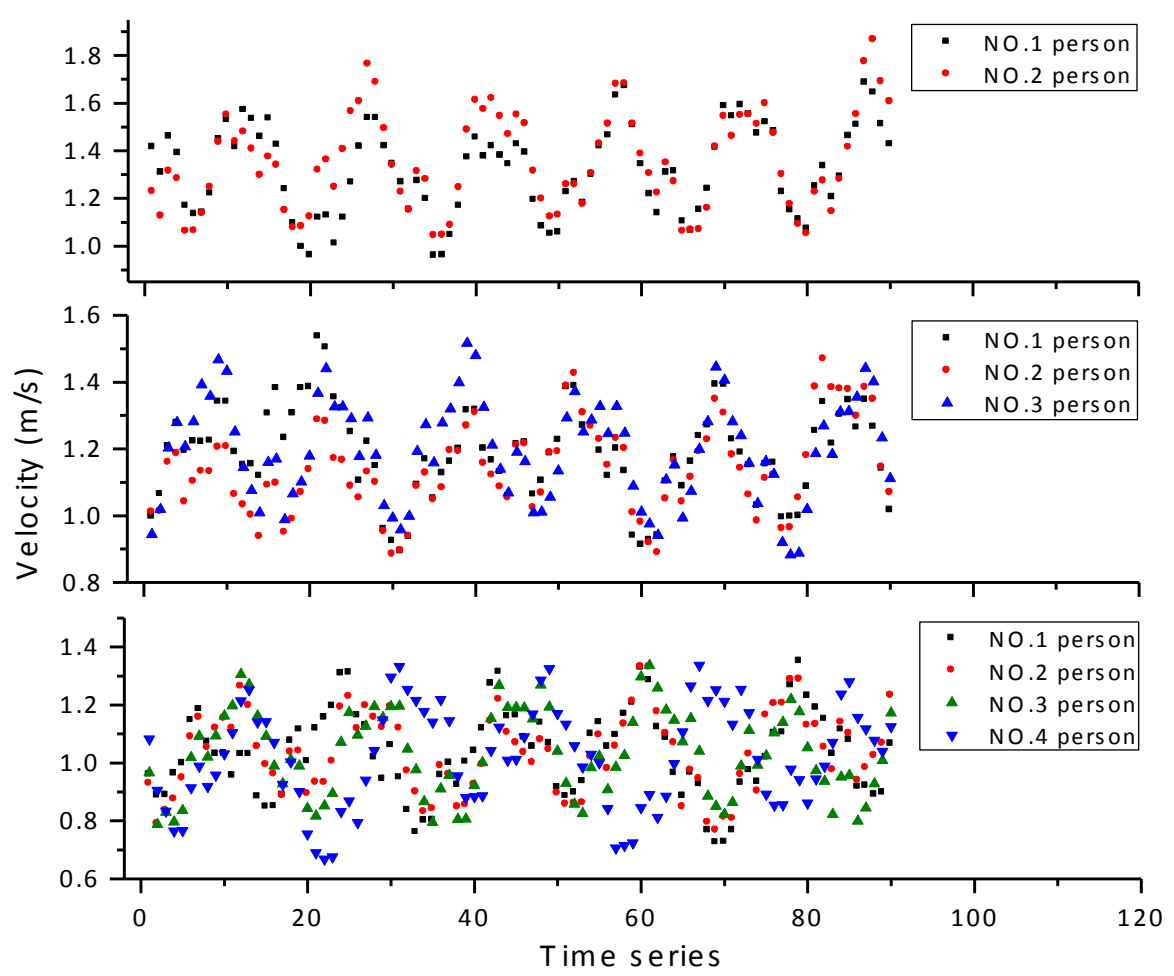

Fig. 8. The time series of group member's velocity of different group size. NO.1 person, NO.2 person, NO.3 person and NO.4 person are the first person, second person, third person and fourth person of the group.

In addition, the pedestrians in the same group have the consistent change trend of the instantaneous velocity. This is result from interactions among several factors, including step frequency, step length and the foot which steps. Though group members' step lengths are different, they have to adjust their step frequencies so as to keep the similar velocity. On the other hand, if one group member steps on the right foot and the other group member steps on the left foot, there occurs lateral collision. Therefore it is hard to keep velocity consistent. This is why the larger the group size is, the harder consistent velocity change emerges and keeps. In addition, the change period is relatively stable, about 15 frames to 20 frames, i.e. $0.5 \mathrm{~s}$ to $0.667 \mathrm{~s}$. It is about $1.5 \mathrm{~Hz}$ to $2 \mathrm{~Hz}$, which is in line with pedestrian's step frequency. Once again this finding shows that the change trend of pedestrian's instantaneous velocity is closely related with pedestrian's walk pattern.

\section{Step Frequency of Group Member}

By the artificial processing method, the time that one pedestrian step is easy to get and we also obtain the pedestrian's step frequency. The Fig. 9 describes each pedestrian group's step frequency. The step frequency of single pedestrian is higher than 2.0 $\mathrm{Hz}$, and some reaches $2.7 \mathrm{~Hz}$, which is a little higher than the experimental data [27]. However, most of pedestrian's step frequency is about $1.9 \mathrm{~Hz}$. Because the group member's movement is affected by each other, the pedestrian with high step frequency has to slow down and achieves the whole group's consistency and comfort. In addition, it is easy to find that the average step frequency, i.e. $2 \mathrm{~Hz}$, is consistent with the velocity change period. However there exist some lower step frequency $(1.5 \mathrm{~Hz})$ and higher step frequency $(2.55 \mathrm{~Hz})$. So the individual difference is obvious. But for the pedestrians in the same group, the step frequency difference is small, that is to say individual difference in the same group is weakened. Because there exits some 
difference among group members, it is natural that their step length is different. In order to keep their velocity consistent, they have to adjust their step frequencies. In addition, with the increasing of the group size, the individual difference in the same group becomes smaller. The reason I infer is that if the step frequency difference of the large group size is too large, some pedestrians are easier to separate from the group.

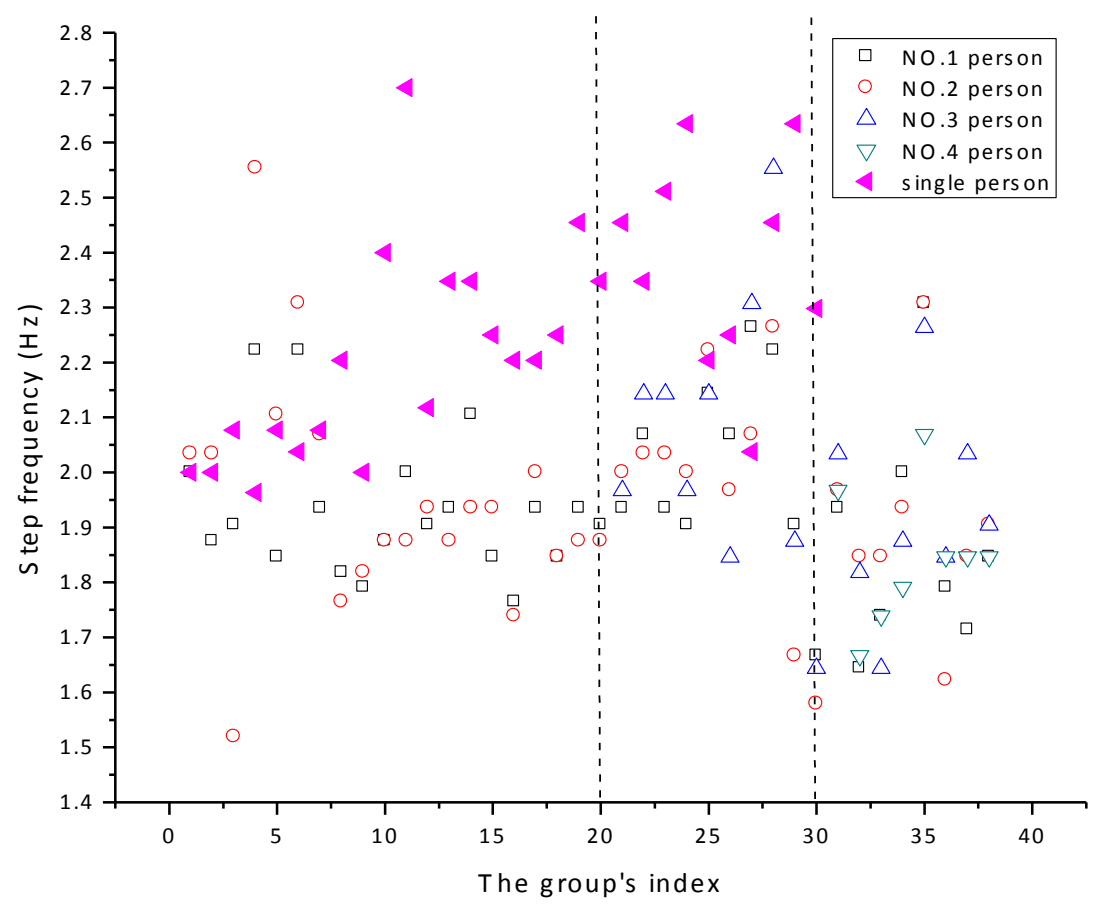

Fig. 9. The step frequency of group member of different group. NO.1 person, NO.2 person, NO.3 person and NO.4 person are the first person, second person, third person and fourth person of the group. And 1-20 are the dyads group, 21-30 are the triads group, 31-38 are the 4 persons groups.

\section{Offset Angle of Group Member}

The velocity and step frequency can reflect group movement consistency from the magnitude point. Thus it is necessary to explore group movement consistency. In order to study the consistency of pedestrian movement direction in the same group, we define a new parameter-offset angle, referring to Eq. 6. As described in Fig. 10, when one pedestrian entered the observed area, he had a start point. And when he left the measurement area, he had an end point. Owing to pedestrian's walking habit or some external factors, it is inevitable that there has some offset in the lateral direction. Therefore, there produces an offset angle during the walking process. It is well accepted that when your friend or partner suddenly changes the movement direction, it is probably that you follow him or her at once. Therefore this parameter is used to illustrate group member's following behavior.

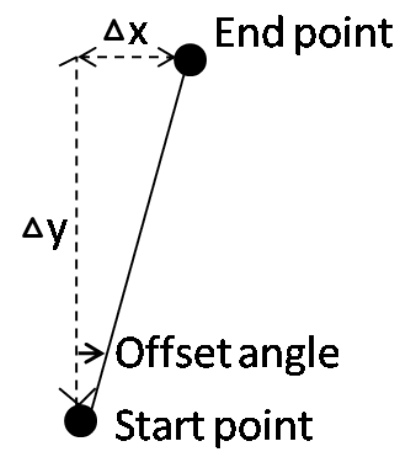

Fig. 10. The definition of pedestrian's offset angle.

Angle $=\arctan \left(\frac{\mid x(i, \text { end })-x(i, \text { start }) \mid}{\mid y(i, \text { end })-y(i, \text { start }) \mid}\right)$ 
Here $\mathrm{x}(\mathrm{i}$,start $), \mathrm{y}(\mathrm{i}$, start $)$ are the $\mathrm{x}$ and $\mathrm{y}$ coordinates of person $\mathrm{i}$ when we start to track, and also $\mathrm{x}(\mathrm{i}, \mathrm{end}), \mathrm{y}(\mathrm{i}$, end $)$ are the $\mathrm{x}$ and $\mathrm{y}$ coordinates of person $\mathrm{i}$ when we end tracking. In fact, this parameter is used to express pedestrian motion gradients.

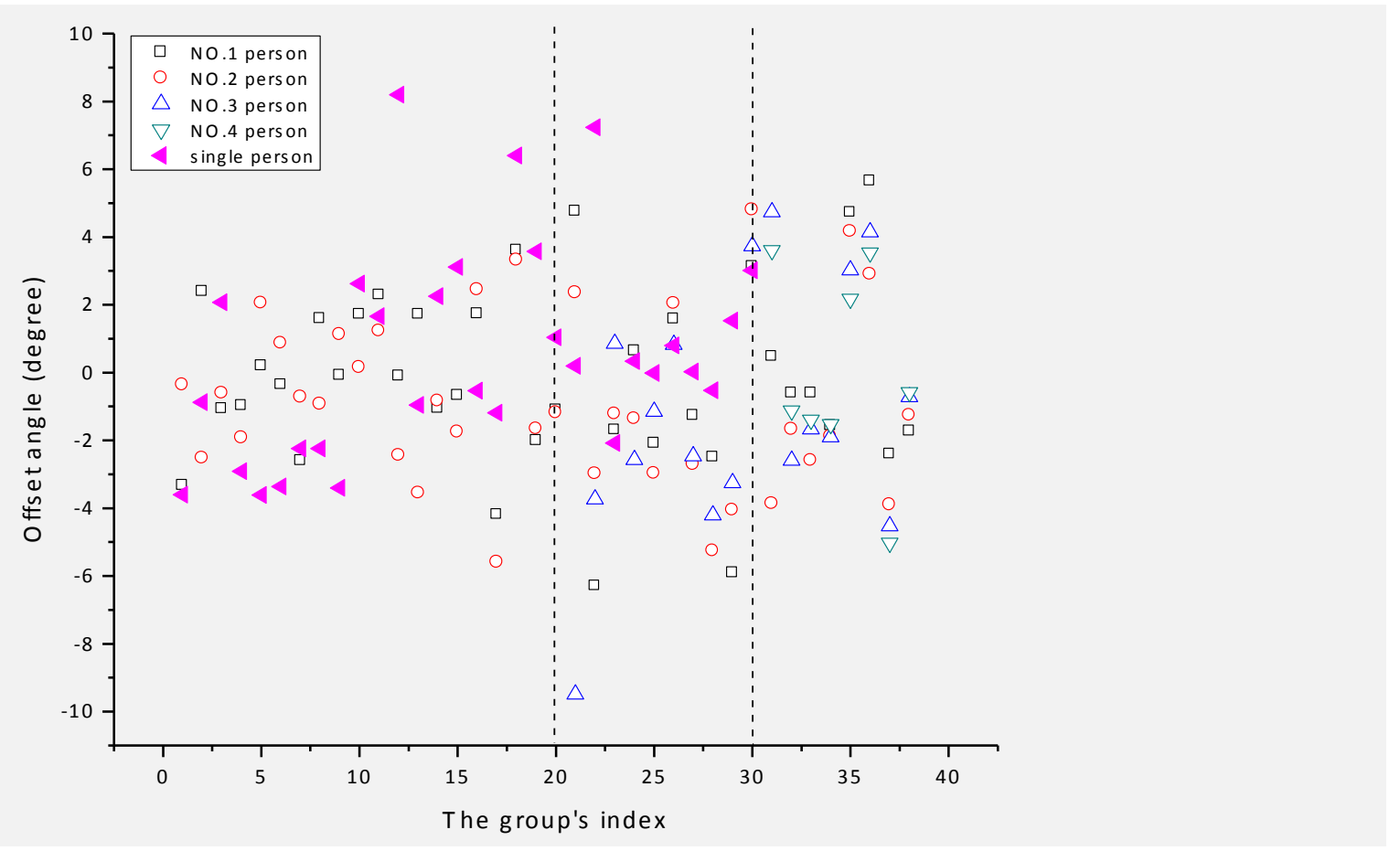

Fig. 11. The offset angle of pedestrian. NO.1 person, NO.2 person, NO.3 person and NO.4 person are the first person, second person, third person and fourth person of the group. And 1-20 are the dyads group, 21-30 are the triads group, 31-38 are the 4 persons groups.

From the above figure, there is minor difference between single pedestrian's offset angle and group member's offset angle. With the group size's increasing, the difference of group member's offset angle in the same group becomes smaller. Because the group members in large group size are easier to separate from other group members their desire to keep together is stronger. The finding shows that the group members have similar offset angle, which illustrates that the group members have a consistent movement direction. With regard to the appropriate value of the offset angle, here it is smaller than 10 degree. It is mainly because that group members walk in the passageway and there is no other attractive factor inducing them to change movement direction. For the large group size, it is found that the pedestrians in the two sides of the group are quite easier to emerge the offset. It results from the relatively large freedom of the pedestrians in the margin region.

\section{SUMMARY}

Social interaction and group coordination are important factors in pedestrian dynamic simulation. While the previous research proves that pedestrian prefers to walk in group, i.e. move with couples, friends or family members. At this time, group members' movement is also affected by the social factor, and is more complex than isolated individual's movement. Owning to the lacking of pedestrian group research, in this manuscript we carried out an observed experiment in a university campus to investigate the movement characteristic of group members. Here we chose 20 groups of male dyads, 10 groups of male triads, 8 groups of four male pedestrians in the freely walking circumstance.

Compared with single pedestrian's movement, the velocity and step frequency of group member is smaller. The reason causing such results includes two aspects, one is the limitation of group member's surrounding space, and the other is the communication among the group members. For different groups, the difference in velocity, step frequency and offset angle is obvious. It mainly stems from the difference of pedestrians. However, for the same group, there has a little diversity in velocity, step frequency and offset angle, which indicates that group members spontaneously adjust their movement overcoming the difference of pedestrians in order to keep the movement consistent. On the other hand, the group members have similar offset angle, and most are smaller than 10 degree. It is mainly because that group members walk in the passageway and there is no other attractive factor inducing them to change movement direction. All these results will be a support for the future modeling research. 


\section{ACKNOWLEDGEMENTS}

This research was supported by National Natural Science Foundation of China (Grant Nos.51178445, 91024025 and 51120165001), the National Basic Research Program of China (No.2012CB719705), the Key Technologies R\&D Program of China During the 12th Five-year Plan Period (Grant Nos.2011BAK07B01, 2011BAK03B02, and 2012BAK13B01) and the Fundamental Research Funds for the Central Universities (No.WK2320000014).

\section{REFERENCES}

[1] M. Chraibi, (2012) Validated force-based modeling of pedestrian dynamics, Forschungszentrum Jülich GmbH, http://kups.ub.uni-koeln.de/4782/.

[2] W. Tian, W. Song, J. Ma, Z. Fang, A. Seyfried, J. Liddle, (2012) Experimental study of pedestrian behaviors in a corridor based on digital image processing, Fire Safety Journal 47: 8-15, http://www.sciencedirect.com/science/article/pii/S0379711211001299.

[3] D. Helbing, P. Molnar, (1995)Social force model for pedestrian dynamics, Phys Rev E 51:4282-4286, http://pre.aps.org/abstract/PRE/v51/i5/p4282_1.

[4] M. Jian, S. Wei-Guo, L. Guang-Xuan, (2010) Multi-grid simulation of pedestrian counter flow with topological interaction, Chinese Physics B 19: 128901, http://iopscience.iop.org/1674-1056/19/12/128901.

[5] D. Helbing, A. Johansson, H.Z. Al-Abideen, (2007) Dynamics of crowd disasters: An empirical study, Phys Rev E 75, http://pre.aps.org/abstract/PRE/v75/i4/e046109.

[6] W.G. Song, Y.F. Yu, B.H. Wang, W.C. Fan, (2006) Evacuation behaviors at exit in CA model with force essentials: A comparison with social force model, Physica A 371: 658-666, http://www.sciencedirect.com/science/article/pii/S0378437106003633.

[7] M. Moussaid, N. Perozo, S. Garnier, D. Helbing, G. Theraulaz, (2010) The walking behaviour of pedestrian social groups and its impact on crowd dynamics, PloS one 5: e10047, http://www.plosone.org/article/info\%3Adoi\%2F10.1371\%2Fjournal.pone.0010047.

[8] I. Karamouzas, M. Overmars, (2012) Simulating and evaluating the local behavior of small pedestrian groups, IEEE Trans Vis Comput Graph 18: 394-406, http://ieeexplore.ieee.org/xpls/abs all.jsp?arnumber=5963666\&tag=1.

[9] F. Qiu, (2010) A Framework for Group Modeling in Agent-Based Pedestrian Crowd Simulations, http://scholarworks.gsu.edu/cs diss/56/.

[10] K.D. Feuz, (2011) Pedestrian Leadership and Egress Assistance Simulation Environment (PLEASE)., UTAH STATE UNIVERSITY, http://digitalcommons.usu.edu/etd/1093/.

[11] A.P. Hare, (1976) Handbook of small group research, http://apps.webofknowledge.com/InboundService.do?

[12] J. James, (1953) The distribution of free-forming small group size, American Sociological Review; American Sociological Review, http://psycnet.apa.org/psycinfo/1954-05814-001.

[13] J.S. Coleman, J. James, (1961) The equilibrium size distribution of freely-forming groups, Sociometry 36-45, http://www.jstor.org/discover/10.2307/2785927?uid=16814080\&uid=364144461\&uid=3737800\&uid=2\&uid=3\&uid=36 3731751\&uid $=67 \&$ uid $=62 \&$ sid $=21102652331793$.

[14] S. Heshka, Y. Nelson, (1972) Interpersonal speaking distance as a function of age, sex, and relationship, Sociometry 491-498,http://www.jstor.org/discover/10.2307/2786529?uid=16814080\&uid=364144461\&uid=3737800\&uid=2\&uid=3 \&uid $=363731751 \&$ uid $=67 \&$ uid $=62 \&$ sid $=21102652331793$.

[15] M. Cook, (1970) Experiments on orientation and proxemics, Human Relations 23: 61-76. http://hum.sagepub.com/content/23/1/61.extract

[16] P. Wirtz, G. Ries, (1992) The pace of life-reanalysed: Why does walking speed of pedestrians correlate with city size?, Behaviour 123: 1-2, http://chinesesites.library.ingentaconnect.com/content/brill/beh/1992/00000123/F0020001/art00005. 
[17] B.A. Barrios, L.C. Corbitt, J.P. Estes, J.S. Topping, (1976) Effect of a social stigma on interpersonal distance, The Psychological Record, http://psycnet.apa.org/index.cfm?fa=search.displayrecord\&uid=1977-08195-001.

[18] H. Singh, R. Arter, L. Dodd, P. Langston, E. Lester, J. Drury, (2009) Modelling subgroup behaviour in crowd dynamics DEM simulation, Applied Mathematical Modelling 33: 4408-4423, http://www.sciencedirect.com/science/article/pii/S0307904X09000808

[19] S.R. Musse, D. Thalmann. (1997) A model of human crowd behavior: Group inter-relationship and collision detection analysis. in Computer Animation and Simulation, http://link.springer.com/chapter/10.1007/978-3-7091-6874-5 3\#page-1

[20] M. Costa, (2010)Interpersonal distances in group walking, Journal of Nonverbal Behavior 34: 15-26, http://link.springer.com/article/10.1007/s10919-009-0077-y\#page-1

[21] F. Qiu, X. Hu, (2010)Modeling group structures in pedestrian crowd simulation, Simulation Modelling Practice and Theory 18: 190-205, http://www.sciencedirect.com/science/article/pii/S1569190X09001555

[22] Z. Wang, B. Song, Y. Qin, L. Jia, (2012) Team-moving effect in bi-direction pedestrian flow, Physica A: Statistical Mechanics and its Applications 391: 3119-3128, http://www.sciencedirect.com/science/article/pii/S0378437112000155

[23] N. Guo, J. Ding, X. Ling, I. Takashi, Q. Shi. (2012) Walking Behavior of Pedestrian Groups in the Merchandise Streets. in CICTP 2012@sMultimodal Transportation Systems-Convenient, Safe, Cost-Effective, Efficient. 2012: ASCE, http://ascelibrary.org/doi/abs/10.1061/9780784412442.067

[24] R. Hartley, A. Zisserman, (2000) Multiple view geometry in computer vision. Vol. 2: Cambridge Univ Press, http://journals.cambridge.org/action/displayFulltext?type=1\&fid=289189\&jid=ROB\&volumeId=23\&issueId=02\&aid=28 $\underline{9188}$

[25] GB10000-88, China national standard human dimensions of Chinese adults. http://www.csres.com/detail/82925.html.

[26] F. Venuti, L. Bruno, (2009) Crowd-structure interaction in lively footbridges under synchronous lateral excitation: A literature review, Phys Life Rev 6, http://www.sciencedirect.com/science/article/pii/S1571064509000244

[27] Z.-M. Fang, W.-G. Song, X. Liu, W. Lv, J. Ma, X. Xiao, (2012) A continuous distance model (CDM) for the single-file pedestrian movement considering step frequency and length, Physica A: Statistical Mechanics and its Applications 391: 307-316, http://www.sciencedirect.com/science/article/pii/S0378437111006376 